\title{
Influencia de las condiciones del secado en el aterronamiento y la compactación del azúcar almacenado
}

\author{
Natalia Castrillón ${ }^{1}$, Benhur Pabón, ${ }^{2}$ Simón Reif-Acherman ${ }^{3 \S}$ \\ ${ }^{1}$ Escuela de Ingeniería Química, Universidad del Valle \\ ${ }^{2}$ Escuela de Ingeniería Química, Universidad del Valle \\ ${ }^{3 \S}$ Escuela de Ingeniería Química, Universidad del Valle, \\ sireache@univalle.edu.co
}

(Recibido: Abril 5 de 2010 - Aceptado: Febrero 15 de 2011)

\begin{abstract}
Resumen
La ocurrencia de fenómenos de aterronamiento y compactación en la industria azucarera debida a factores internos controlables de las últimas etapas del proceso de producción, aleatorios externos al mismo, o una combinación de ambos, ocasiona significativos perjuicios operacionales y económicos. Un estudio de la optimización de las condiciones de operación manipulables en la operación de secado permite minimizar la ocurrencia de dichos fenómenos. Utilizando como principal criterio de evaluación los diferentes efectos en el tiempo sobre la humedad final de los cristales almacenados, los resultados obtenidos permitieron identificar las magnitudes más apropiadas de cantidad y temperatura de entrada del aire alimentado al secador instalado en la refinería de un ingenio de la región del Valle del Cauca, para cada una de las condiciones ambientales típicas de las tres jornadas de producción diarias. Verificaciones estadísticas de la implementación de normas de proceso formuladas con base en las conclusiones del estudio revelan la obtención de cristales con un contenido de humedad final por debajo del máximo limite de especificación permitido del $0.05 \%$, la practica eliminación del fenómeno de compactación en dos de las tres jornadas, y reducciones significativas del aterronamiento en todos los casos.
\end{abstract}

Palabras Clave: Aterronamiento, Compactación, Humedad, Temperatura, Flujo, Secador rotatorio, Azúcar refinada.

CHEMICAL ENGINEERING

\section{Influence of drying conditions on the caning and compactation of stored sugar}

\begin{abstract}
The occurrence of caking and compacting phenomena in the sugar industry, because of controllable internal factors of the concluding steps of the production process, random external factors, or to a combined of both types, causes significant financial and operative damages. A study on the optimization of the controllable operation conditions in the drying step allows minimizing these inconveniences. By using the evaluation of the different effects on the stored crystals final moisture with the time as the main evaluation criteria, the results allowed the identification of the more convenient magnitudes of flow rate and temperature of the air introduced to the dryer installed in the sugar refinery of a mill of the region of Valle del Cauca for each one of the typical ambient conditions of the three daily production shifts. Statistical testing of a established rule based on the conclusion's study reveal the obtaining of crystals with a final moisture content below the maximum specified limit of $0.05 \%$, the almost full elimination of the compression phenomena in two of the three shifts, and significant decreasing of caking in all situations.
\end{abstract}

Keywords: Caking, Compression, Moisture, Temperature, Rotary dryer, Refined sugar. 


\section{Introducción}

La aglomeración espontanea de cristales de azúcar es un fenómeno de frecuente ocurrencia en las etapas de almacenamiento y transporte de azúcar refinado (Bagster, 1970a, 1970b), debido principalmente a su alta sensibilidad a las condiciones ambientales del medio que lo rodea. El contenido interno de agua de los cristales suele liberarse, bajo condiciones normales, al cabo de 24 horas después del procesamiento en fábrica, período de tiempo necesario establecido para el acondicionamiento del producto antes de empacarse y transportarse. Esta situación se refleja en la obtención de humedades próximas a las correspondientes al equilibrio. Las magnitudes de estas humedades características están directamente relacionadas con los valores de humedad relativa y temperatura tanto del aire utilizado en el proceso de secado, como de aquel presente en la bodega de almacenamiento y empaque.

Es muy común, sin embargo, que debido a la alta higroscopicidad del producto y factores diversos tales como las altas presiones a las que se somete el azúcar en los arrumes, largos periodos de contacto estático, elevadas humedades relativas del aire en la zona de almacenamiento, y variaciones bruscas de temperatura (debidas a cambios horarios o modificaciones en las corrientes de aire) algunas migraciones de humedad continúen sucediéndose aun después del empaque. Este incremento en el contenido de agua promueve el fenómeno de compactación, identificado por procesos de ablandamiento o plastificación de los cristales, en las cuales sus superficies externas se humedecen debido a la presencia del agua. La creación de una interfase cristalina y puentes líquidos entre los cristales de azúcar conduce a formación de conglomerados de alta dureza, incapaces de fluir libremente, y difíciles de separar, los cuales forzan su reprocesamiento o una conversión en azucares de menor calidad. Si la fragilidad del conglomerado es menor, y puede lograrse una recuperación de la fluidez del producto por medios mecánicos, el evento se denomina de aterronamiento. La compactación y aterronamiento de azúcar refinada, a granel o empacada, en circunstancias de extensos periodos de almacenamiento, con altos volúmenes de producción y grandes arrumes de azúcar en las bodegas, aumenta significativamente las devoluciones de azúcar y el trabajo de recuperación del producto terminado. Esta situación repercute obviamente en forma de pérdidas de eficiencia y capacidad de producción, deterioro de la imagen comercial, y sobrecostos para las industrias involucradas.

Investigaciones previas en el tema muestran que el tamaño de los cristales y la humedad relativa del aire con él que estos entran en contacto, son dos de los principales parámetros responsables de esta situación, mientras que la temperatura de almacenamiento, para el caso a granel (Ramphal, 1989), el arreglo de los sacos, e incluso las especificaciones del material especifico de las bolsas utilizadas, para el caso de producto empacado (Excell \& Mellet, 1986), pueden también constituirse en elementos a considerar. Experimentalmente se ha verificado que la presencia de partículas finas (inferiores a $250 \mu \mathrm{m}$ ) de azúcar en el proceso propician la ocurrencia del fenómeno a humedades relativas incluso tan bajas como el 33 \% (Rogé \& Mathlouthi, 2000). La forma específica de los cristales en este rango especifico de tamaños afecta decididamente la posibilidad de fluidez libre del producto (Rogé \& Mathlouthi, 2003). El efecto de la variación de las condiciones del aire ha resultado más complejo de evaluar por las interacciones con otras variables, constituyéndose en el objeto de investigaciones más detalladas (Billings \& Paterson, 2008, Billings et al, 2006, Christakis et al, 2006, Mathlouthi \& Roge, 2003).

El tamaño del grano de azúcar no es, sin embargo, la única causa de compactación y aterronamiento atribuible al proceso en sí mismo. La humedad de los cristales es obviamente también afectada por procesos de secado incompletos, o excesivamente rápidos, caracterizados los primeros por excesiva cantidad de agua en los cristales, y los segundos por encapsulamiento de una humedad que posteriormente migrará a la superficie. Sea cual sea el escenario, el entendimiento de la forma en 
que el azúcar interacciona con la humedad del aire resulta esencial para la prevención de los fenómenos anteriormente citados (Mathlouthi \& Roge, 2004, 2003).

En décadas recientes, varios estudios teóricos relacionados con el funcionamiento de secadores rotatorios han sido llevados a cabo con miras a optimizar la prevención de los fenómenos de compactación y aterronamiento del azúcar. Buena parte de estos desarrollos incluyen programas complejos de simulación aplicables a diferentes tipos de sólidos para la predicción de contenidos de humedad y temperatura de salida de los cristales en función del tiempo y una longitud característica del secador (Gopinathan et al, 2006; Cao \& Langrish, 2000).

Programas específicos para secadores de azúcar, utilizando balances de materia y energía tanto bajo la suposición de equilibrio termodinámico (Douglas et al, 1993) como para la situación real de no-equilibrio (Wang et al, 1993, 1995), han aparecido de manera casi simultánea con estudios que plantean la formación de películas de jarabe en la superficie de los cristales (Tait et al, 1994; BenYoseph, \& Hartel, 1999, 2006) y la evidente presencia de azúcar amorfo en las mismas a ciertas condiciones particulares de secado (Meadows, 1993; Thompson, 1998; van der Poel et al, 1998). El muy posible incremento significativo del grado de supersaturacion del jarabe debido a altas velocidades de evaporación, especialmente en el llamado periodo de velocidad decreciente de secado, se traduce en un desbalance de agua en la capa superficial de los cristales, el cual no alcanza a compensarse con el proceso de cristalización, promoviendo la formación de una capa de este azúcar amorfo.

La mayor capacidad de adsorción de agua por parte de esta capa ha sido considerada por varios investigadores un elemento responsable a considerar en los fenómenos de aglomeración en las etapas de almacenamiento y acondicionamiento de azúcar (Bruijn \& Marijnissen, 1996; Meadows, 2000). No obstante contribuir significativamente al entendimiento de los diferentes y complejos procesos que se suceden en la etapa de secado, ninguno de este tipo de estudios provee al operario de procesos de las herramientas prácticas necesarias para ajustar aquellas variables manipulables asociadas con la variación de las condiciones ambientales con miras a mantener las características principales del azúcar a enviarse a la etapa de acondicionamiento.

Si bien las condiciones de trabajo de la secadora han sido incorporadas desde un punto de vista práctico en programas de investigación integrales de la situación como algunas de las principales causas de los fenómenos de compactación y aterronamiento del azúcar (Ferreira, 2006), ningún estudio detallado parece haberse realizado en este campo considerando exclusivamente esta etapa del proceso y la incidencia de las características ambientales de una industria en particular.

El objetivo de este trabajo se centró en la identificación de las magnitudes óptimas de las principales variables manipulables en la operación de la etapa de secado en orden a prevenir la ocurrencia de formación de aglomerados. La investigación, llevada a cabo en uno de los ingenios de mayor capacidad de producción de azúcar refinada de la región del Valle del Cauca (INCAUCA, S.A), permitió el establecimiento de protocolos específicos de trabajo para las diferentes condiciones ambientales típicas imperantes en la región geográfica de localización del ingenio.

\section{Metodología}

El modelo de secadora/enfriadora utilizada en el estudio comprende dos secciones principales: una primera etapa en donde el producto proveniente de las centrifugas, con un contenido de humedad 
variable $(0.5-1.5 \%)$, se pone en contacto con aire caliente en un sistema co-corriente, la cual es seguida de un enfriamiento realizado en contracorriente con aire ambiente externo. Las condiciones teóricas del producto de salida son $0.035 \%$ de humedad y una temperatura en el rango de 25 a $40{ }^{\circ} \mathrm{C}$. El equipo está dotado de un sistema interno de panales y placas deflectoras que suavizan la caída de los cristales, previniendo el riesgo de ruptura debido al movimiento rotatorio del tambor y el cambio súbito de temperatura al pasar de una sección a otra. Unos dispositivos internos permiten igualmente el retiro con el aire caliente del polvillo generado por la fractura de los cristales tanto en el sistema de transporte previo como en el interior del equipo, el cual es posteriormente recolectado por lavado del aire con agua caliente $\mathrm{y}$ retornado al proceso. Las especificaciones del equipo y sus condiciones de operación se resumen en la Tabla 1.

De acuerdo con la infraestructura técnica experimental disponible se escogieron la temperatura y el flujo de aire de entrada a la secadora como las principales variables a estudiar en la cuantificación del efecto sobre el contenido de humedad final del azúcar y su porcentaje de aterronamiento y compactación. El estudio completo se basó en una cantidad promedio constante representativa del ingenio particular de 23.6 ton/h de flujo de entrada de azúcar a la secadora, correspondiente a la operación con un sistema de tres centrifugas. La determinación del rango de temperaturas a estudiar se basó en estudios teóricos ampliamente conocidos de comportamiento del secado del azúcar, mientras que la de las tres cifras de flujo de aire utilizado, resultantes de los balances de materia y energía alrededor de la etapa de secado, correspondió a las limitaciones prácticas de posibilidades de posicionamiento del dámper del ventilador y el historial de fabricación. Se identificaron igualmente tres jornadas diferentes diarias representativas de las variables condiciones ambientales imperantes en la región geográfica del estudio. La Tabla 2 ilustra los diferentes rangos analizados para cada una de estas variables.

El diseño experimental así diseñado incluyo entonces un total de 15 tratamientos ( 5 niveles de temperatura del Aire x 3 niveles de flujo de aire) aplicables a cada uno de los niveles del factor de condición ambiental. El diseño de bloques utilizado fue completamente al azar, con un

Tabla 1. Especificaciones de la secadora y condiciones de operación.

\begin{tabular}{|c|c|}
\hline Variable & Valor \\
\hline Velocidad del tambor, rev/min & 4.3 \\
\hline Angulo de inclinación, ${ }^{\circ}$ & 2.28 \\
\hline Pendiente & 0.04 \\
\hline Relación longitud / diámetro, (L/D) & 7.61 \\
\hline Tiempo promedio de paso, $\mathrm{min}$ & 11.34 \\
\hline Velocidad de la válvula rotativa de entrada, rpm & $18.4-18.6$ \\
\hline Flujo de aire de entrada, $\mathrm{m}^{3} / \mathrm{h}$ & 70421.4 \\
\hline Flujo másico experimental de azúcar de entrada a la secadora, ton $/ \mathrm{h}$ & 26.3 \\
\hline Flujo másico de polvillo, $\mathrm{kg} / \mathrm{h}$ & 79.26 \\
\hline Flujo másico de terrones, $\mathrm{kg} / \mathrm{h}$ & 159.02 \\
\hline
\end{tabular}

Tabla 2. Rangos estudiados para cada una de las variables

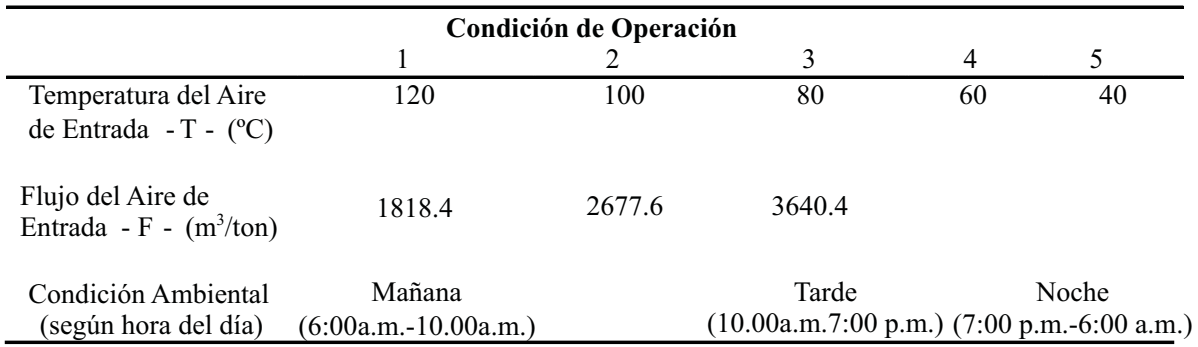


arreglo en parcelas subdivididas, recurriendo como criterio de bloqueo a las semanas en las cuales se efectuaron las mediciones de las variables de respuesta.

Las características del arreglo fueron las siguientes:

$\begin{array}{ll}\text { Parcela Mayor: } & \text { Condición Ambiental } \\ \text { Subparcela: } & \text { Flujo del Aire } \\ \text { Subsubparcela: } & \text { Temperatura del Aire } \\ \text { Número de Bloques: } & \text { Tres }\end{array}$

El modelo asociado con el tipo de diseño y arreglo experimental escogido fue entonces:

$$
\begin{aligned}
& Y_{\mathrm{ijk} 1}=\mu+S_{\mathrm{i}}+J_{\mathrm{j}}+\varepsilon_{\mathrm{ij}}+F_{\mathrm{k}}+(J \times F)_{\mathrm{jk}}+\varepsilon_{\mathrm{ijk}}+T_{1}+ \\
& (J \times T)_{\mathrm{j} 1}+(F \times T)_{\mathrm{kl}}+(J \times F \times T)_{\mathrm{jk} 1}+\varepsilon_{\mathrm{ijk} 1}
\end{aligned}
$$

en donde $Y$ representa la variable de respuesta, $=$ el promedio general, y $S, J, F$ y $T$ los efectos individuales debidos al bloque, la condición ambiental, el flujo de aire, y la temperatura, respectivamente. Los términos con paréntesis identifican efectos de tres interacciones binarias y una ternaria, mientras que $=$ los errores asociados con las interacciones indicadas por los subíndices de cada término específico.

Las variables de respuesta escogidas fueron:

$$
\begin{aligned}
& \% \text { Aterronamiento }=\frac{\text { Bultos aterronados }}{45 \text { bultos }} \times 100 \\
& \% \text { Compactación }=\frac{\text { Bultos compactados }}{45 \text { bultos }} \times 100
\end{aligned}
$$

La evaluación del contenido de humedad se llevó a cabo utilizando el método gravimétrico estándar:

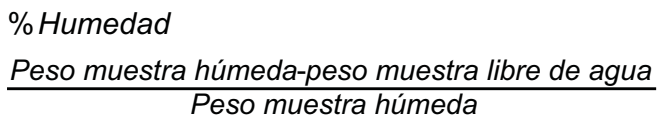

La determinación de los porcentajes de aterronamiento y compactación se realizó sobre la base de lotes con el número definido de bultos acomodables en una estiba, es decir 45 , cada uno con $50 \mathrm{~kg}$ de azúcar. Los muestreos correspondientes se llevaron a cabo en la tercera, cuarta, y quinta semanas de permanencia de los bultos en la bodega, trasladándolos de la estiba en donde se encontraban a otra vacía, y utilizando el sentido del tacto para el monitoreo de identificación de las unidades aterronadas y compactadas. Los resultados obtenidos fueron analizados a la luz de análisis de varianza (ANOVA) y pruebas de promedios de grupos de Duncan para las diferentes variables de respuesta, utilizando para ello el paquete estadístico S.A.S, versión 8.2. (Tabla 4)

\section{Resultados y discusión}

La Tabla 3 resume los resultados promedio de los balances de materia y energía para los diferentes tratamientos seleccionados en cada jornada.

Tabla 3. Magnitudes promedio de las variables resultantes de balances de materia y energía

\begin{tabular}{lrrr}
\hline Variable & \multicolumn{1}{c}{ Mañana } & \multicolumn{1}{c}{ Tarde } & \multicolumn{1}{c}{ Noche } \\
\hline Agua retirada por el aire de secado, $(\mathrm{kg} / \mathrm{h})$ & 347.9 & 53.8 & 162.5 \\
Flujo másico de vapor de agua $\mathrm{M}_{\mathrm{V}}(\mathrm{kg} / \mathrm{h})$ & 2497.1 & 677.0 & 1181.4 \\
Transferencia de calor del vapor al aire $\mathrm{Q}_{\mathrm{V}},(\mathrm{kJ} / \mathrm{h})$ & 5365781.8 & 1451825.8 & 2538132.3 \\
Transferencia de calor del aire al sólido $\mathrm{Q}_{\mathrm{S}},(\mathrm{kJ} / \mathrm{h})$ & 691111.2 & 579720.1 & 988676.3 \\
Eficiencia Energética en el Secado ${ }_{\mathrm{S}},(\%)$ & 26.5 & 45.4 & 51.9 \\
\hline
\end{tabular}


Tabla 4. Análisis de varianza para las variables de respuesta asociadas con el mejoramiento de la etapa de secado

\begin{tabular}{|c|c|c|c|c|c|c|}
\hline \multirow{2}{*}{ Fuentes de variación } & \multirow{2}{*}{ GLI } & \multicolumn{5}{|c|}{$\begin{array}{r}\text { Cuadrado medio } \\
\end{array}$} \\
\hline & & \multicolumn{2}{|c|}{ Compactación } & \multicolumn{2}{|c|}{ Aterronamiento } & Humedad \\
\hline Semana & 2 & 0.89 & $*$ & 4560.59 & $*$ & 0.026 \\
\hline Condición ambiental & 2 & 1.89 & ** & 1711.66 & $\mathrm{~ns}$ & 0.000 \\
\hline Flujo & 2 & 0.03 & ns & 380.48 & ns & 0.107 \\
\hline Condición ambiental x Flujo & 4 & 0.38 & ns & 1516.22 & $* *$ & 0.099 \\
\hline Temperatura & 4 & 0.86 & $* *$ & 1143.25 & $* *$ & 0.044 \\
\hline Temperatura x Flujo & 8 & 1.41 & $* *$ & 1379.07 & $* *$ & 0.069 \\
\hline Condición ambiental x Temperatura & 8 & 0.74 & $* *$ & 1608.02 & $* *$ & 0.056 \\
\hline Condición amb. X Temperatura x Flujo & 16 & 0.64 & $* *$ & 2851.78 & $* *$ & 0.039 \\
\hline Promedio ( \%) & & 1. & & 56.32 & & 0.0568 \\
\hline CV $(\%)$ & & & & 24.3 & & 14.7 \\
\hline
\end{tabular}

** Diferencias significativas al 1\%, *Diferencias significativas al $5 \%$, ns Diferencias no significativas

\subsection{Incidencia individual de las variables}

Un análisis separado del efecto de cada una de las variables consideradas sugiere algún tipo de tendencias tanto alrededor del fenómeno de compactación como del de aterronamiento, si bien la dirección y órdenes de magnitud de las influencias en ambos fenómenos no resultan similares. Las Figuras 1 a 4 y 5 a 8 presentan la incidencia separada de cada variable en los dos fenómenos y el comportamiento del contenido de humedad en cada caso, respectivamente.

El tiempo, por ejemplo, parece tener mayor incidencia en el fenómeno de aterronamiento que en el de compactación, ya que mientras el aumento en el primero de los casos se dio en un rango de 45 a $70 \%$, el orden de magnitud de la disminución en el segundo fue de 2.5 a 1.5 , aproximadamente (Figura 1). Estos resultados pueden explicarse a la luz del incremento con el tiempo de las dificultades de separación entre los cristales asociadas con el fortalecimiento de los puentes líquidos entre ellos, y el simultáneo desmoronamiento de los terrones inicialmente formados, debido a su fragilidad. Así mismo, la violación del límite superior de especificación de humedad (L.S.E.H.) del azúcar de $0.05 \%$ (no obstante haber salido del equipo con indicadores apropiados para esta misma variable), y la generación de problemas de compactación a partir de la cuarta semana de almacenamiento evidencian la ocurrencia de alguno(s) de los previamente mencionados problemas de secado.

De otro lado, y como era de esperarse, los mayores resultados de compactación mostrados en las horas de la noche (del orden del $4.0 \%$ ), con respecto a aquellos observados en la mañana, indican una asociación directa del fenómeno con los porcentajes de humedad relativa imperantes en cada rango horario.

La utilización de flujos de aire elevados y consecuentes velocidades de secado excesivamente altas permiten un tiempo de contacto insuficiente para la liberación del agua de los granos de azúcar, promoviendo un encapsulamiento parcial inicial de ésta, su posterior migración a la superficie del cristal, y la generación de la compactación, tal y como se aprecia en la Figura 3.

El análisis de la incidencia de la temperatura del aire de entrada en los fenómenos de aglomeración es tal vez el más complejo de todos, presentando comportamientos opuestos para compactación y aterronamiento por ejemplo a temperaturas intermedias (alrededor de $80^{\circ} \mathrm{C}$ ). Los resultados 


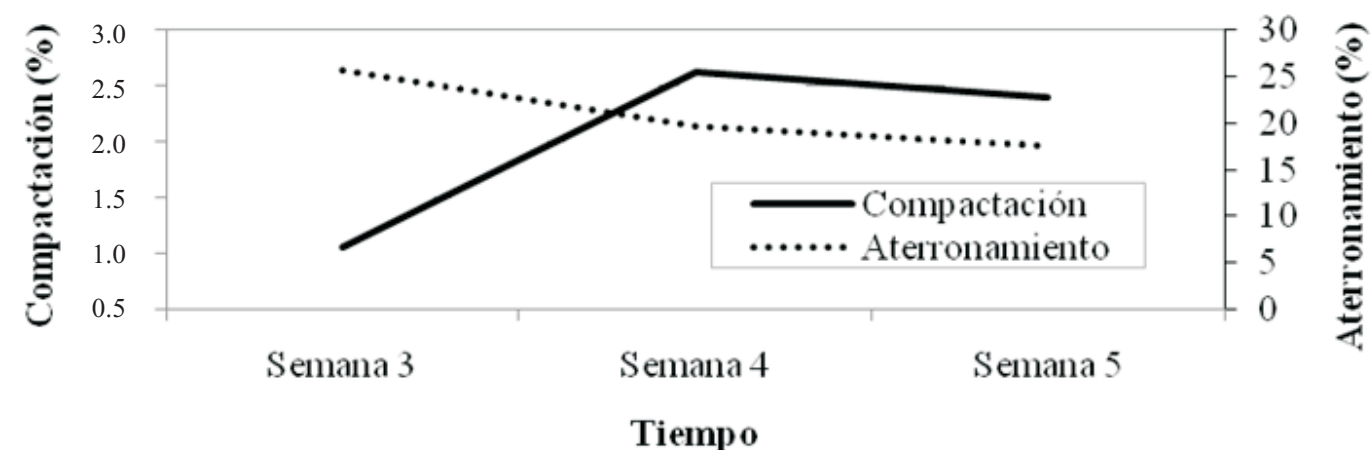

Figura 1. Incidencia de la variable individual tiempo en los fenómenos de aglomeración

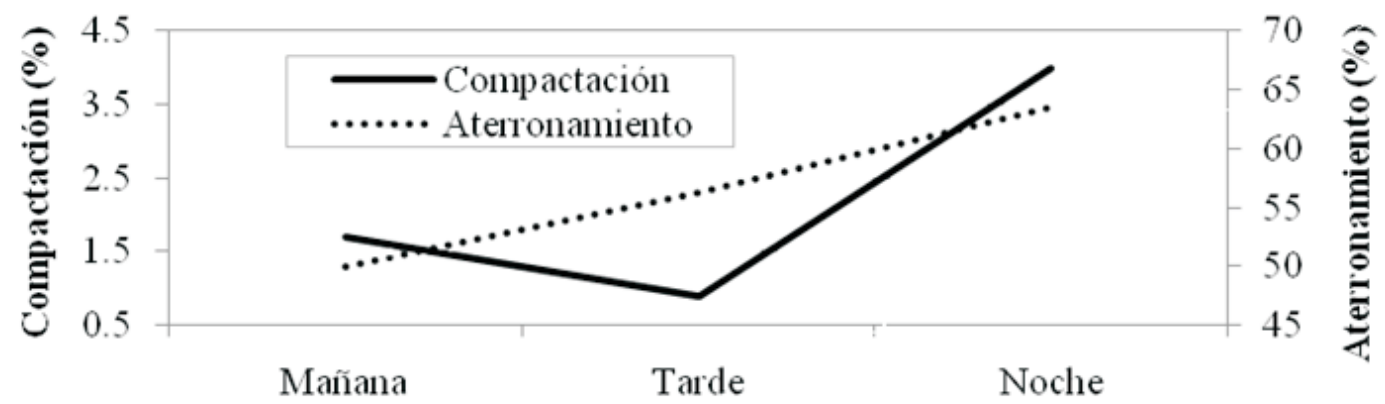

\section{Periodehorario}

Figura 2. Incidencia de la variable individual periodo horario en los fenómenos de aglomeración

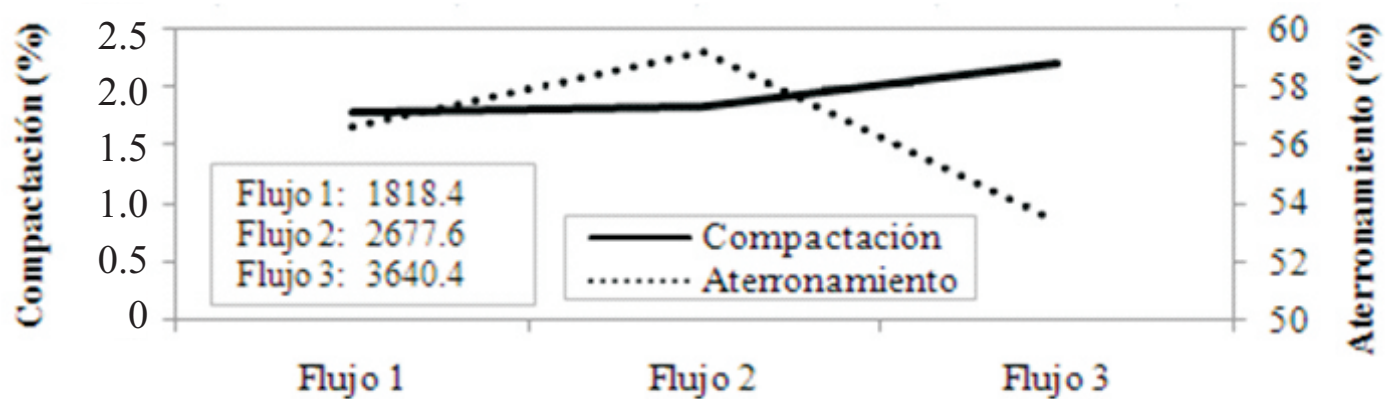

Flujo de entrada de aire a la secadora ( $\mathrm{m}^{3} /$ ton)

Figura 3. Incidencia de la variable individual flujo de entrada de aire en los fenómenos de aglomeración 


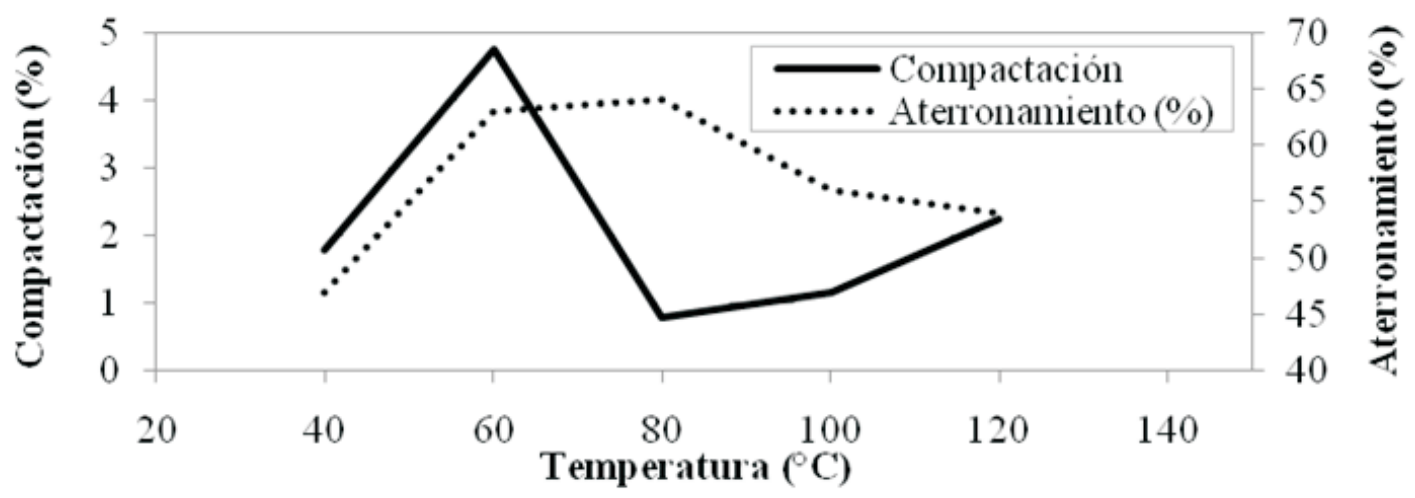

Figura 4. Incidencia de la variable individual temperatura en los fenómenos de aglomeración

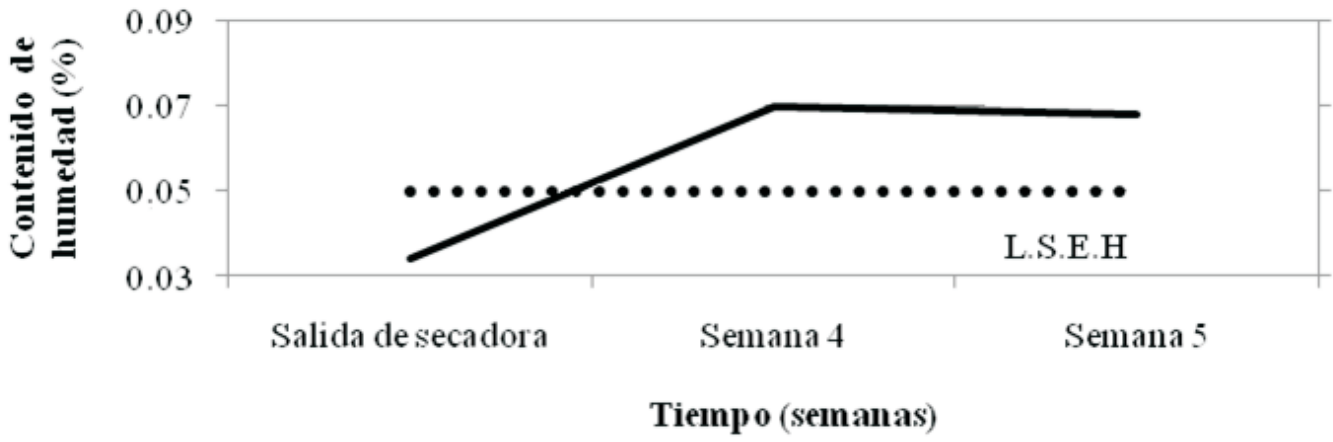

Figura 5. Incidencia de la variable individual tiempo en el contenido de humedad del azúcar

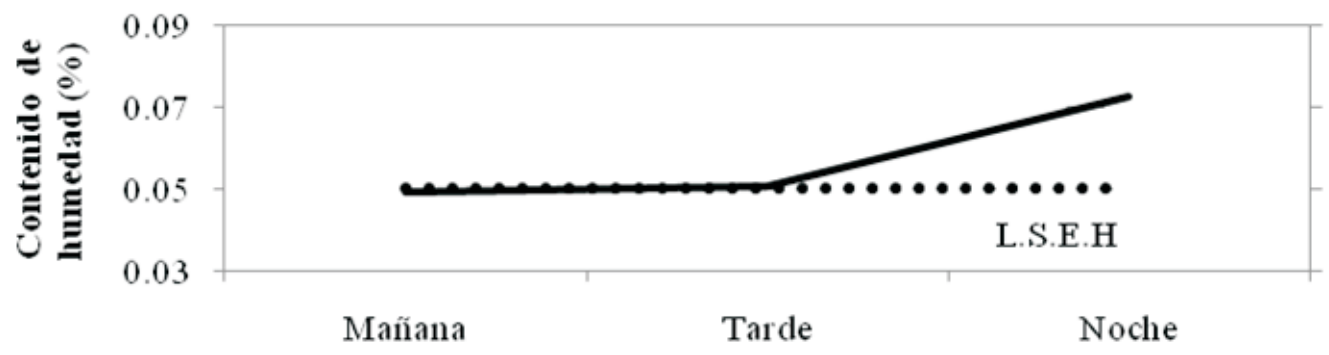

Periodo horario

Figura 6. Incidencia de la variable individual periodo horario en el contenido de humedad del azúcar 


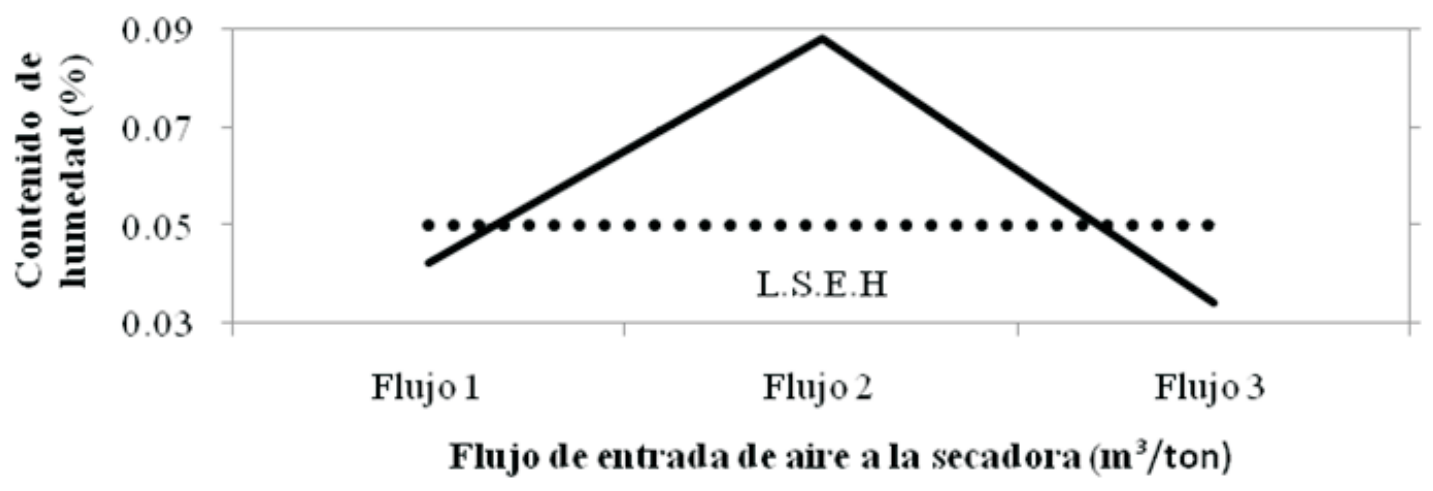

Figura 7. Incidencia de la variable individual flujo de entrada de aire en el contenido de humedad del azúcar

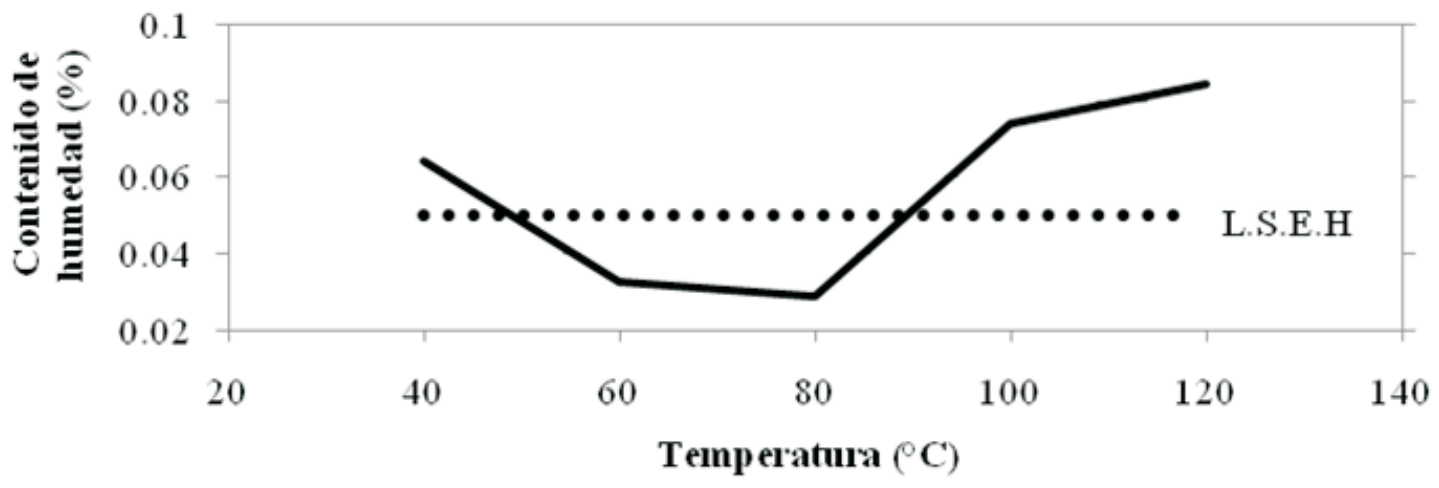

Figura 8. Incidencia de la variable individual temperatura en el contenido de humedad del azúcar

obtenidos de promoción de la compactación de los cristales en ambos extremos del rango de temperaturas estudiado concuerdan con lo esperado cualitativamente de las teorías de migración de agua en las diferentes etapas del secado. En rangos bajos $\left(40 \quad 60^{\circ} \mathrm{C}\right)$, la falta de garantías para adecuados procesos de secado debido a comparativamente bajos niveles de transferencia de calor y materia, explica fácilmente el grado de disminución de humedad de los cristales por debajo de los niveles requeridos para su almacenamiento. Cuando por el contrario la temperatura del aire de entrada es alta, la del empaque del azúcar también resulta serlo, y la ya aceptada teoría de la formación de una capa de azúcar amorfo rodeando los cristales mencionada en la sección de introducción determina un retardamiento de la parte concluyente del secado.

\subsection{Incidencia de interacciones de múltiples variables}

Se estudiaron los efectos de las interacciones de parejas de variables que, según el análisis de varianza, mostraron diferencias significativas en el comportamiento de las tres variables de respuesta. La diferenciación de resultados experimentales para cada periodo horario resultó evidente, tal y como se muestra en las Figuras 9 a 11, 12 a 14, y 15 a 17, para las jornadas de la mañana, tarde, y noche, respectivamente. El análisis del efecto de la interacción de las condiciones ambientales de temperatura y flujo de aire de entrada, tanto sobre los propios fenómenos de compactación y aterronamiento, como sobre el máximo contenido de humedad del producto, permitió la selección del tratamiento más apropiado para cada una de las jornadas estudiadas. 


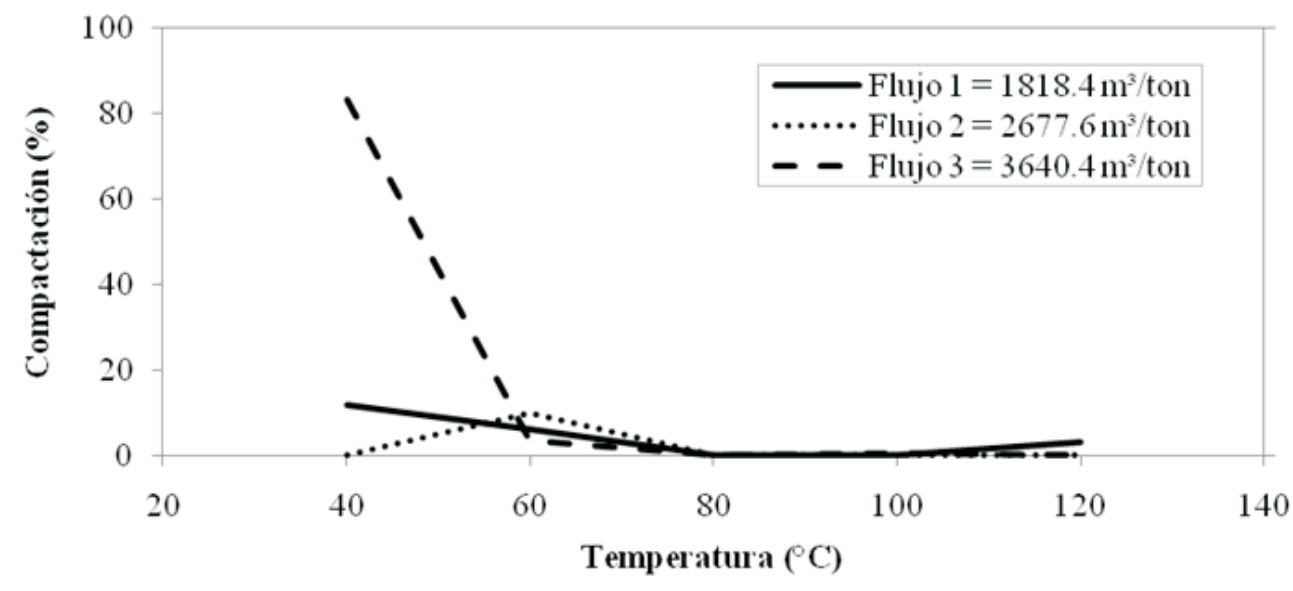

Figura 9. Efecto de la interaccion flujo x temperatura en la compactación (periodo de la mañana)

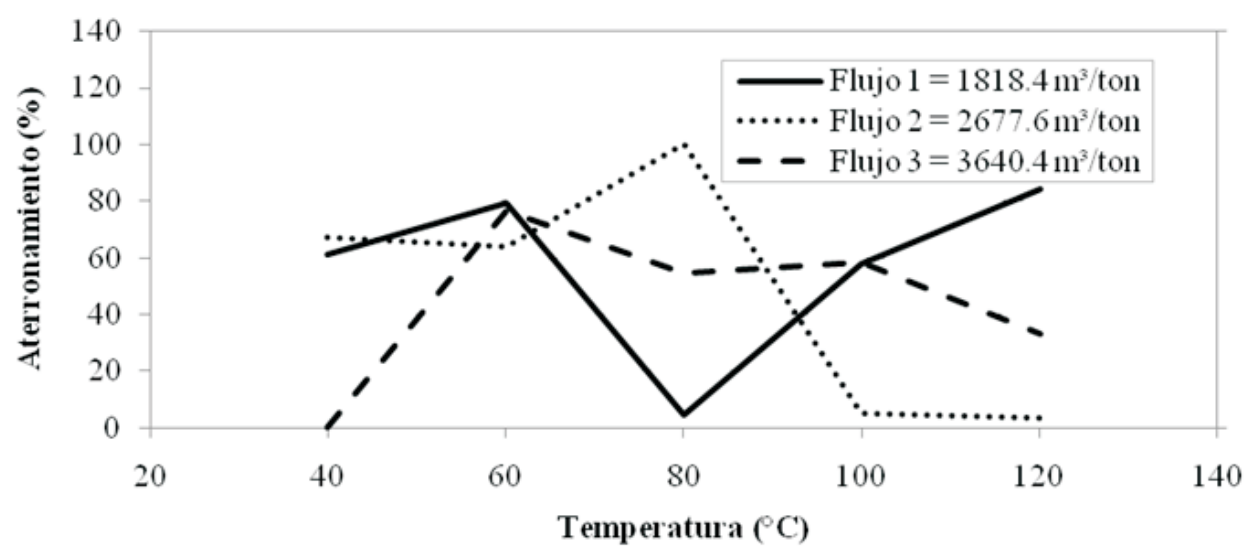

Figura 10. Efecto de la interaccion flujo x temperatura en el aterronamiento ( periodo de la mañana)

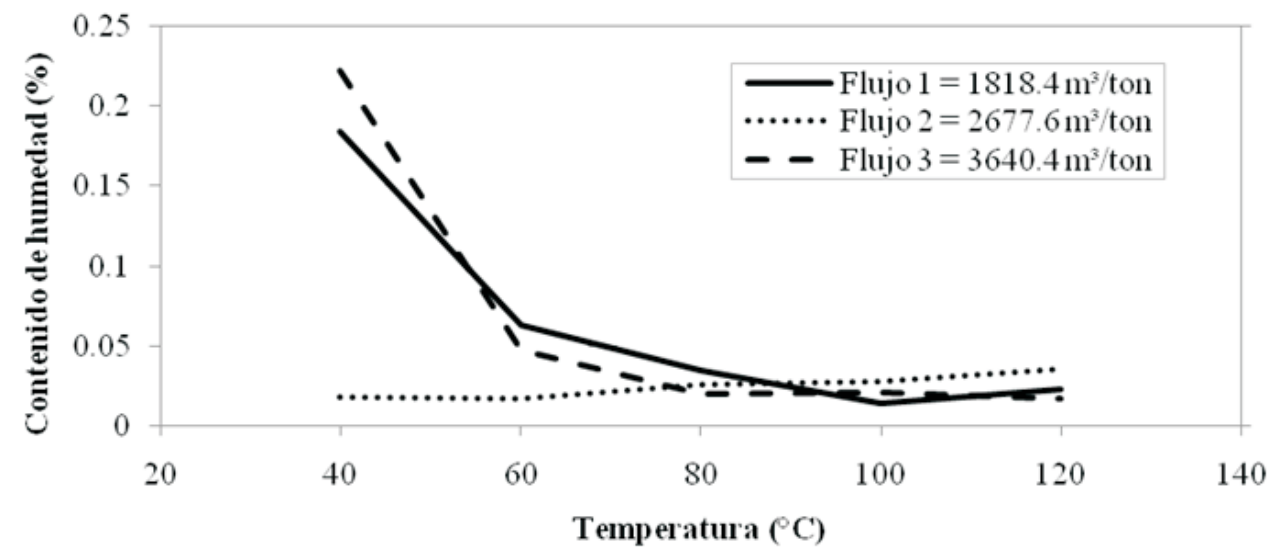

Figura 11. Efecto de la interaccion flujo x temperatura en el contenido de humedad (periodo de la mañana) 


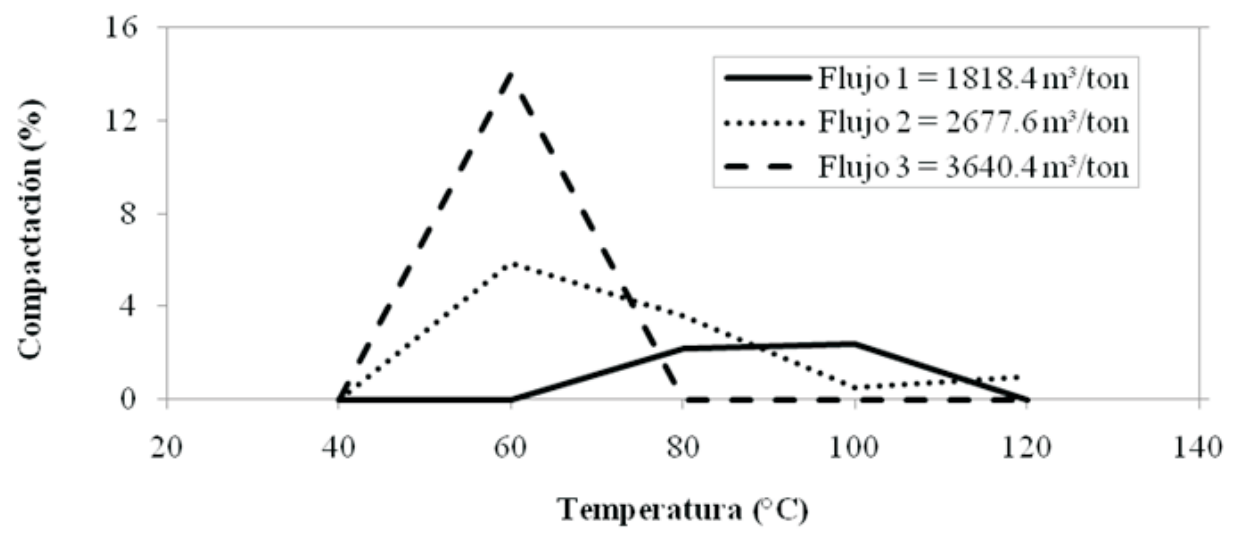

Figura 12. Efecto de la interaccion flujo x temperatura en la compactación (periodo de la tarde)

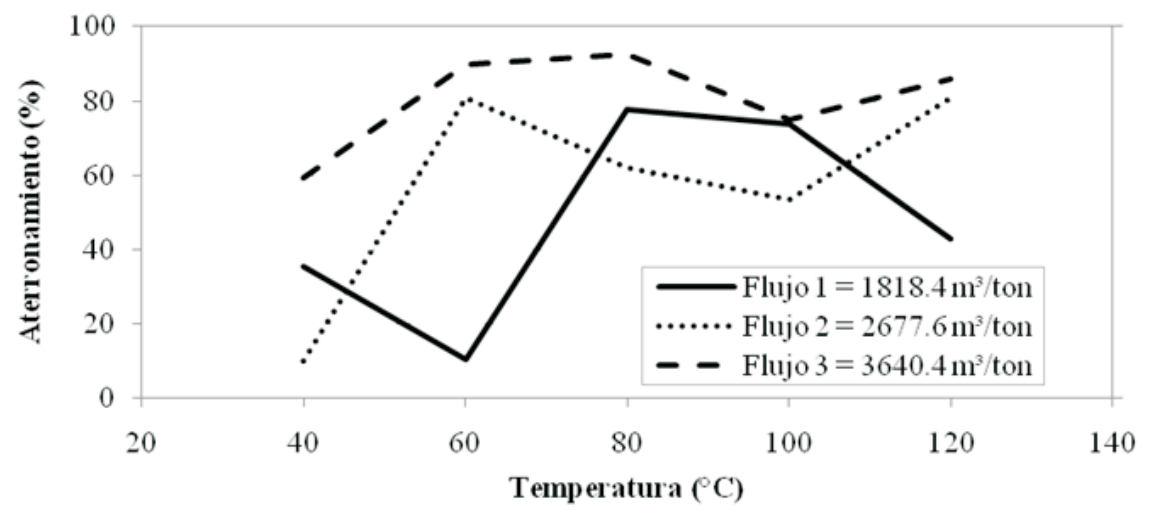

Figura 13. Efecto de la interaccion flujo x temperatura en el aterronamiento ( periodo de la tarde)

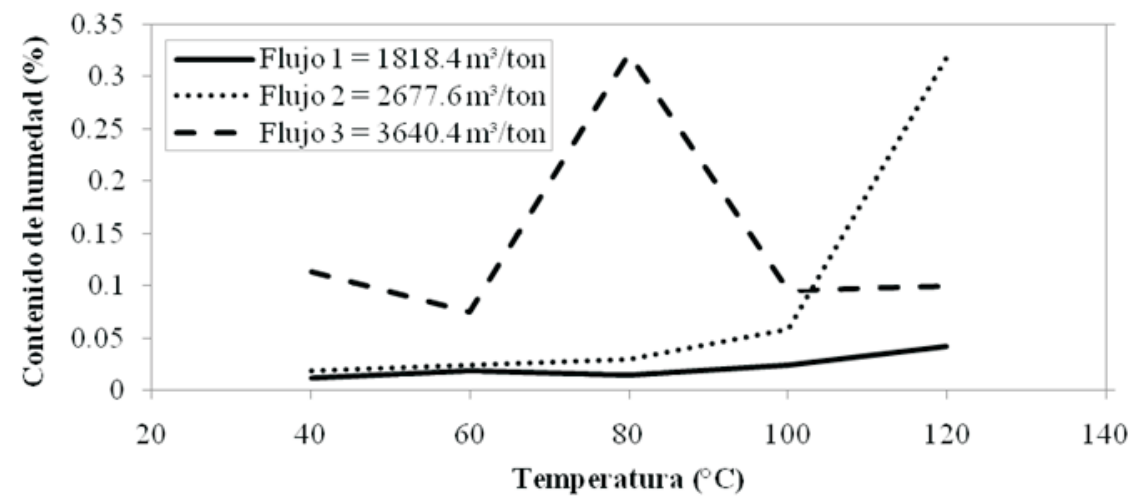

Figura 14. Efecto de la interaccion flujo x temperatura en el contenido de humedad (periodo de la tarde) 


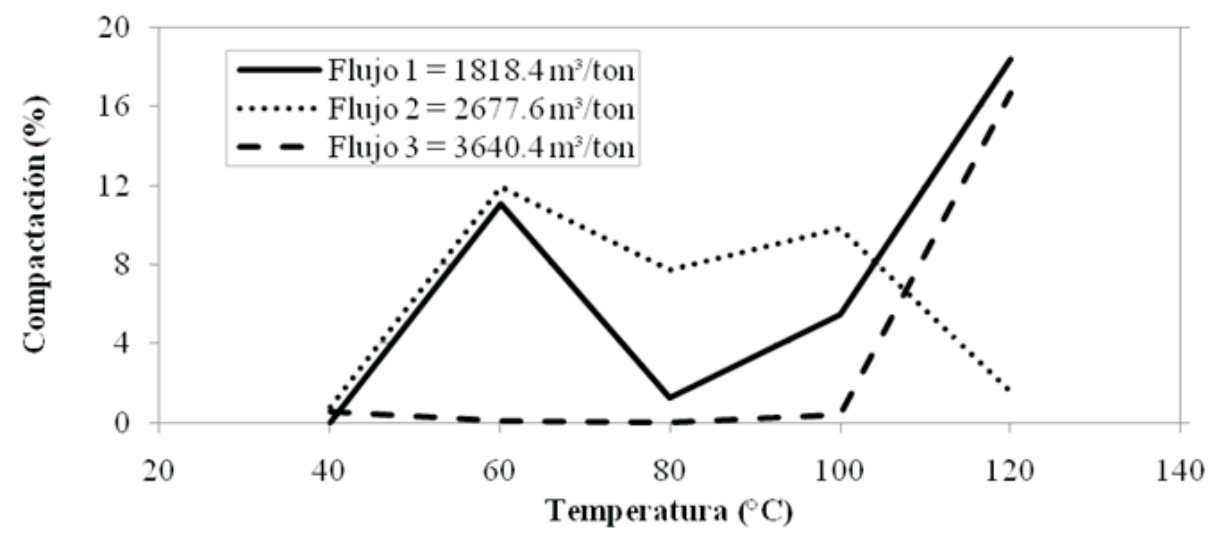

Figura 15. Efecto de la interaccion flujo x temperatura en la compactación (periodo de la noche)

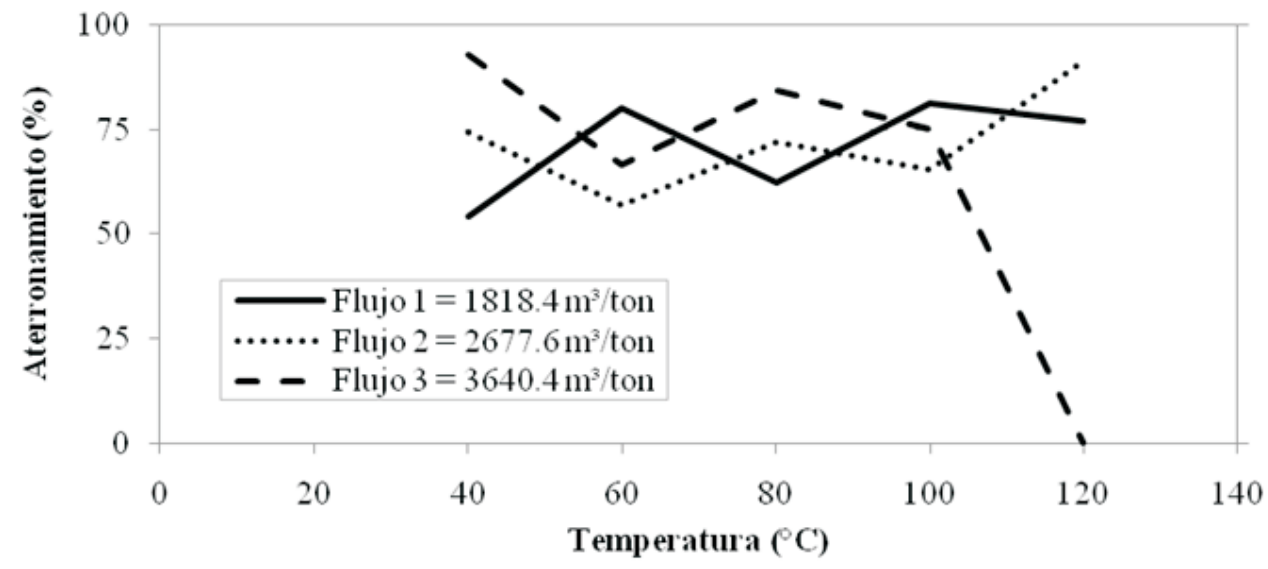

Figura 16. Efecto de la interaccion flujo x temperatura en el aterronamiento (periodo de la noche)

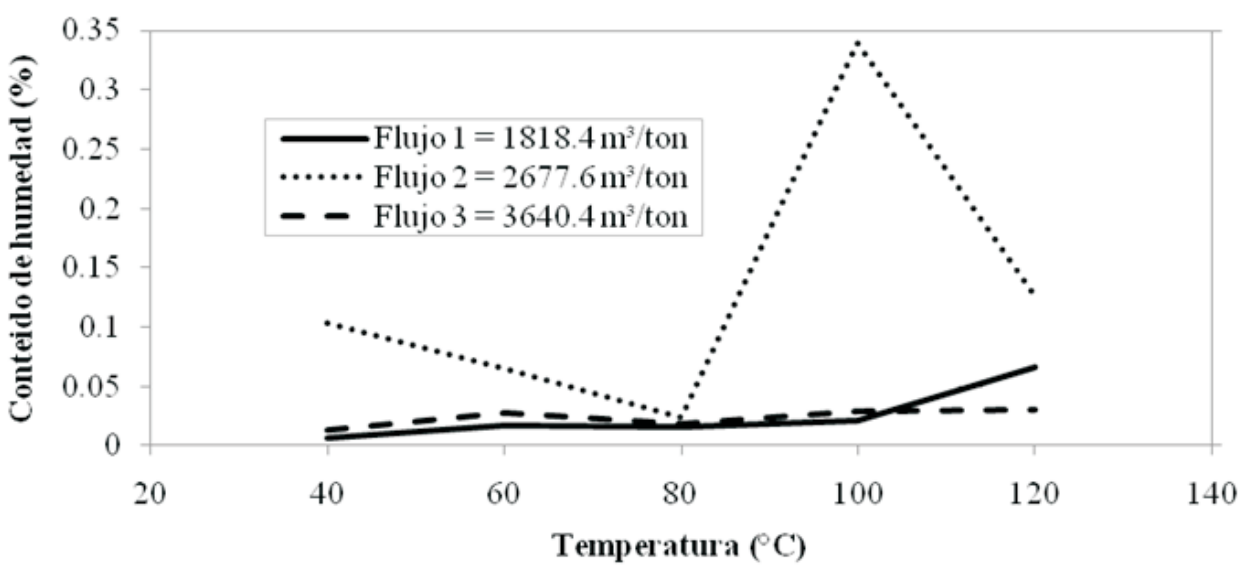

Figura 17. Efecto de la interaccion flujo x temperatura en el contenido de humedad (periodo de la noche) 
Estas jornadas han sido convenientemente adaptadas en términos de los rangos promedio de los dos principales parámetros indicadores de cada una, temperatura y humedad relativa, para propósitos de generalizar las conclusiones alcanzadas. La Tabla 5 resume estas condiciones.

\section{Conclusiones}

Varios factores inciden en la ocurrencia con el tiempo de los fenómenos de compactación y aterronamiento del azúcar almacenado. La tradicional creencia, por ejemplo, de que este tipo de fenómenos es causado exclusivamente por los excesivos pesos a los cuales el azúcar es sometido cuando se lo arruma en las bodegas de azúcar terminado, debido a la creación de elevados esfuerzos cortantes y grandes presiones, es falsa. Comprobaciones experimentales que debieron hacerse en este aspecto como parte de la presente investigación permitieron incidentalmente mostrar que en algunas ocasiones bultos colocados en la parte inferior de la estiba no presentaban signos de compactación o formación de terrones, mientras que otros, ubicados en la parte superior de la misma estiba y con ningún o a lo sumo solo unos cuantas unidades encima, y expuestos a las mismas condiciones generales, si mostraban señales de aterronamiento.

La investigación permitió igualmente verificar la incidencia de otro elemento generalmente asociado con los mismos fenómenos, relacionado con las características del material de empaque. Bultos que no contenían la usual capa interna de protección (liner) en su material de empaque no presentaron ni compactación ni aterronamiento debido a la liberación de humedad hacia el ambiente por los poros del empaque de polipropileno. Esta situación, sin embargo, es inconveniente, debido a que la no presencia de esta capa aumenta el riesgo de contaminación del azúcar.

Las condiciones ambientales son parámetros determinantes en la ocurrencia de los fenómenos de compactación y aterronamiento del azúcar almacenado. La apropiada consideración de sus variables representativas, temperatura y humedad relativa, permite elaborar normas de proceso fácilmente aplicables por parte de operarios de capacitación estándar, lográndose una incidencia directa en la optimización de la etapa de secado. $\mathrm{Su}$ implementación en campo es sencilla, y requiere simplemente de un higrómetro debidamente protegido del polvillo, la curva de calibración del damper del ventilador, y un sencillo sistema de control de temperatura del aire de entrada acoplado con la fuente de suministro de vapor.

Si bien el estudio realizado demostró la eficiencia de la optimización de la etapa de secado en la prevención de fenómenos de aglomeración del azúcar almacenado, es claro que un control completo de los mismos requiere del acompañamiento de condiciones físicas adicionales de diferente orden que contribuyan a garantizar condiciones adecuadas de almacenamiento, tales como barreras que eviten la presencia de corrientes de aire promotoras de gradientes de temperatura, o la formación de arrumes con excesivas cargas estáticas.

Tabla 5. Parámetros óptimos establecidos de trabajo según condiciones ambientales imperantes

\begin{tabular}{lcc}
\hline Periodo horario & Temperatura aire entrada $\left({ }^{\circ} \mathrm{C}\right)$ & Flujo aire entrada $\left(\mathrm{m}^{3} /\right.$ ton $)$ \\
\hline T ambiente $: 25-29^{\circ} \mathrm{C}$ & 100 & 2677.6 \\
$\begin{array}{l}\text { Humedad relativa }: 65-80 \% \\
\text { T ambiente }: 30-35^{\circ} \mathrm{C}\end{array}$ & 60 & 1818.4 \\
Humedad relativa $: 45-70 \%$ & & \\
T ambiente $: 25-29{ }^{\circ} \mathrm{C}$ & 60 & 3640.4 \\
Humedad relativa $: 60-90 \%$ & & \\
\hline
\end{tabular}




\section{Agradecimientos}

Los autores desean expresar sus agradecimientos a las directivas y personal de planta de Incauca S.A. e Incauca Refinería de Colombia por toda la colaboración prestada para la realización de este trabajo.

\section{Referencias bibliográficas}

Bagster, D.F., 1970a. Cause, prevention and measurement of the caking of refined sugar a review. Part I. International Sugar Journal 72 (861), 263-267.

Bagster, D.F., 1970b. Cause, prevention and measurement of the caking of refined sugar a review. Part II. International Sugar Journal 72 (862), 298-302.

Ben-Yoseph, E., \& Hartel, R. W. (1999). Computer modeling of sugar crystallization during drying of thin sugar films. Journal of Crystal Growth 198(199), 1294-1298.

Ben-Yoseph, E., \& Hartel, R. W. (2006). Computer simulation of sugar crystallization in confectionery products. Innovative Food Science and Emerging Technologies 7 (3), 225-232.

Billings, S.W., Bronlund, J.E. \& Paterson, A.H.J. (2006). Effects of capillary condensation on the caking of bulk sucrose. Journal of Food Engineering 77 (4), 887-895.

Billings, S.W. \& Paterson, A.H.J. (2008). Prediction of the onset of caking in sucrose from temperature induced moisture movement. Journal of Food Engineering 88 (4), 466-473.

Bruijn, J. M. de, \& Marijnissen, A. A. W. (1996). Moisture content of sugar crystals and influence of storage conditions. In Proceedings of the sugar processing research conference, April 1417 (pp. 183-197). New Orleans, Louisiana, USA.

Cao, W. F., \& Langrish, T. A. G. (2000). The development and validation of a system model for a countercurrent cascading rotary dryer. Drying Technology 18(1), 99-115.
Christakis, N., Wang, J., Patel, M.K., Bradley, M.S.A., Leaper, M.C. \& Cross, M. (2006). Aggregation and caking processes of granular materials: continuum model and numerical simulation with application to sugar. Advanced Powder Technology 17 (5), 543-565.

Douglas, P. L., Kwade, A., Lee, P. L., \& Mallick, S. K. (1993). Simulation of a rotary dryer for sugar crystalline. Drying Technology 11 (1), 129-155.

Excell, T.L. \& Mellet, P. (1986). Refined sugar storage trials using various types of $25 \mathrm{~kg}$ paper bags. Proceedings of The South African Sugar Technologists' Association 60, 112-115.

Ferreira, M.S., 2006. Experiencias en el control de la humedad y aterronamiento del azucar refinado en el ingenio Risalralda S.A.. En Memorias del VII Congreso de la Asociación Colombiana de Técnicos de la Caña de Azúcar, Cali, Colombia, 2, 214-217.

Gopinathan, N., Johanson, K.D., Lin, C.L. \& Miller, J.D. (2006). Systems Approach to Solution of Caking Problems. In Proceedings of the 2006 AIChE Spring National Meeting and 5th World Congress on Particle Technology, Orlando, USA, paper 231b.

Mathlouthi, M. \& Rogé, B. (2003). Water vapour sorption isotherms and the caking of food powders. Food Chemistry 82 (1), 61-71.

Mathlouthi, M. \& Rogé, B. (2004). Caking of white sugar and how to prevent it. In Proceedings of the 78th Annual Congress of South African Sugar Technologists' Association, Mount Edgecombe, South Africa, 495-503.

Meadows, D. M. (1993). Somewhat dry A new look at the conditioning of refined sugar. Proceedings of the South African Sugar Technologists' Association, 67, 160-165.

Meadows, D. M. (2000). Refined sugar drying, conditioning, and storage. In C. C. Chou (Ed.), Handbook of sugar refining. A manual for the design and operation of sugar refining facilities (pp. 245292). New York: Wiley. 
Ramphal, R.R. (1989). The effect of sugar temperature on the operating performance of a conditioning silo. Proceedings of The South African Sugar Technologists' Association 63, 6467.

4Rogé, B. \& Mathlouthi, M. (2000). Caking of sucrose crystals: effect of water content and crystal size. Zuckerindustrie 125 (5), 336-339.

Rogé, B. \& Mathlouthi, M. (2003). Caking of white crystalline sugar. International Sugar Journal 105 (1251), 128-136.

Tait, P. J., Schinkel, A. L., \& Greig, C. R. (1994). The development and application of a generalized model for sugar drying. In Proceedings of the ninth international drying symposium IDS'94, Vol. A (pp. 203-211). Gold Coast, Australia.

Thompson, P. (1998). Drying, cooling and bulk storage of sugar. International Sugar Journal 100, 223-230.

Van der Poel, P. W., Schwieck, H., \& Schwartz, T. (1998). Sugar technology. Beet and cane sugar manufacture (Drying and cooling of sugar, pp. 867-880). Berlin: Bartens.

Wang, F. Y., Cameron, I. T., \& Litster, J. D. (1995). Further theoretical studies on rotary drying processes represented by distributed systems. Drying Technology 13 (3), 737-751.

Wang, F. Y., Cameron, I. T., Litster, J. D., \& Douglas, P. L. (1993). A distributed parameter approach to the dynamics of rotary drying processes. Drying Technology, 11 (7), 1641-1656. 